\title{
New 3-hydroxy-2-naphthoic hydrazide derivatives: thiosemicarbazides and 1,2,4-triazole-3-thiones, their synthesis and in vitro antimicrobial evaluation
}

\author{
Lukasz Popiołek $^{1} \cdot$ Kinga Paruch $^{1} \cdot$ Paweł Patrejko $^{1} \cdot$ Anna Biernasiuk $^{2} \cdot$ \\ Monika Wujec ${ }^{1}$
}

Received: 25 January 2016 / Accepted: 10 June 2016 / Published online: 17 June 2016

(C) The Author(s) 2016. This article is published with open access at Springerlink.com

\begin{abstract}
In this paper, we describe twelve 3-hydroxy2-naphthoic hydrazide derivatives (six thiosemicarbazides and six 1,2,4-triazole-3-thiones) which we have synthesized. Thiosemicarbazide derivatives were prepared by condensation reaction of 3-hydroxy-2-naphthoic hydrazide with appropriate isothiocyanates. The 1,2,4-triazole3-thiones were synthesized by the cyclization reaction of corresponding thiosemicarbazide derivatives in alkaline media. All obtained compounds have been analyzed by ${ }^{1} \mathrm{H}$ NMR and ${ }^{13} \mathrm{C}$ NMR spectra and evaluated for in vitro antimicrobial activity against a panel of reference strains of microorganisms, including Gram-positive bacteria, Gramnegative bacteria and fungi belonging to yeasts Candida spp. Our antimicrobial screening results indicated that some of the newly obtained compounds showed high antibacterial activity, especially against Gram-positive bacteria. Among them, the activity of compounds $\mathbf{1 , 2}$ and $\mathbf{4}$ was moderate to strong against staphylococci. In addition to this, compounds $\mathbf{1}$ and $\mathbf{2}$ showed two times higher activity than cefuroxime and four times higher activity than ampicillin against Bacillus cereus ATCC 10876.
\end{abstract}

Electronic supplementary material The online version of this article (doi:10.1007/s13738-016-0911-1) contains supplementary material, which is available to authorized users.

Łukasz Popiołek

lukasz.popiolek@umlub.pl

1 Department of Organic Chemistry, Faculty of Pharmacy, Medical University of Lublin, 4A Chodźki Street, 20-093 Lublin, Poland

2 Department of Pharmaceutical Microbiology, Faculty of Pharmacy, Medical University of Lublin, 1 Chodźki Street, 20-093 Lublin, Poland

\section{Graphical Abstract}
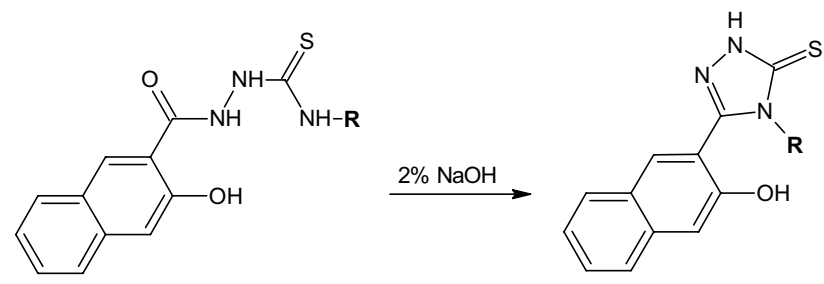

$\mathbf{R}=\mathrm{Alk}, \mathrm{Ar}$

Keywords 3-Hydroxy-2-naphthoic hydrazide . 1,2,4-Triazole derivatives $\cdot$ Thiosemicarbazide derivatives . Antimicrobial activity $\cdot$ MIC $\cdot$ MBC

\section{Introduction}

The development of drug resistance to clinically used antiinfective agents reveals that there is an urgent need for the discovery of new antimicrobial compounds to treat multiresistant infections [1]. Considering this, our attention focused on thiosemicarbazide and 1,2,4-triazole derivatives, which have been incorporated into a wide range of therapeutically interesting molecules and possess many medical applications [2].

It is known that thiosemicarbazides act as antibacterial [3-5], antifungal [6, 7], antitubercular [8], anticonvulsant $[9,10]$ and antitumor agents $[11,12]$. In turn, the 1,2,4-triazoles display a broad range of biological activities, such as antibacterial [13-19], antifungal [20-22], antitubercular [23-26], antiviral [27] and antiproliferative properties [2830]. Several compounds possessing the triazole scaffold are clinically useful medicines, such as ribavirin [31], terconazole [32] or fluconazole [33]. 
Looking for the interesting starting material for new research project, our group found 3-hydroxy-2-naphthoic hydrazide, which caught our attention. The choice of 3-hydroxy-2-naphthoic hydrazide as a starting material for our synthesis was based on the literature findings concerning this hydrazide. This hydrazide is very useful of itself in the histochemical detection of peroxidation in animal tissues. According to Pompella and Comporti [34], 3-hydroxy-2-naphthoic hydrazide/Fast Blue B reaction is a sensitive and reliable tool for the revelation of lipid peroxidation-derived carbonyl compounds in tissue. Pompella and Comporti [34] suggested that in the tissues of heterogeneous cell composition its use can allow the approach of choice for the recognition of the cell types involved in the lipid peroxidation process in vivo. Dogan et al. [35] using this hydrazide, synthesized novel thiosemicarbazides, 1,2,4-triazoles and 1,3,4-thiadiazoles with significant antibacterial activity against Staphylococcus aureus, Escherichia coli and Pseudomonas aeruginosa, as well as antifungal activity against Candida albicans. Similarly, Khalil et al. [36] obtained benzenobenzo[g]phthalazine-1,4-dione derivatives from this hydrazide, which showed interesting antibacterial activity against Bacillus thuringiensis and E. coli, comparable to commonly used chemotherapeutics like ampicillin and chloramphenicol. In another study, 1,2,4-triazoles derived from this hydrazide showed promising in vitro anticancer activity against 60 human tumor cell lines [37].

As a continuation of our research interest in the field of synthesis and antimicrobial screening of heterocyclic compounds, we have undertaken this research, in which we have synthesized and test for in vitro antimicrobial activity a series of thiosemicarbazides and 1,2,4-triazole-3-thiones obtained form 3-hydroxy-2-naphthoic hydrazide. This seemed reasonable in the view of the above mentioned facts.

\section{Experimental}

\section{Chemistry}

All required chemicals and solvents were purchased from Sigma-Aldrich (Munich, Germany) and Merck Co. (Darmstadt, Germany) and used without further purification. Melting points were determined with Fisher-Johns blocks (Fisher Scientific, Germany) and are uncorrected. The ${ }^{1} \mathrm{H}$ NMR spectra were recorded on a Bruker Avance 300 apparatus (Bruker BioSpin GmbH, Germany) in DMSO- $d_{6}$ with TMS as internal standard. The ${ }^{13} \mathrm{C}$ NMR spectra were recorded on a Bruker Avance 300 apparatus in DMSO. The chemical shifts are expressed on the $\delta$ (ppm) scale using TMS as the standard reference. The coupling constants $(J)$ are given in Hertz. Spin multiples are given as s (singlet), d (doublet), dd (doublet of doublets), $\mathrm{t}$ (triplet), q (quartet), $\mathrm{m}$ (multiplet) and $\mathrm{b}$ (broad). The progress of the reaction and purity of obtained compounds were monitored by TLC, using pre-coated aluminum sheets plates 60 F254 (Merck Co. USA) in a $\mathrm{CHCl}_{3} / \mathrm{C}_{2} \mathrm{H}_{5} \mathrm{OH}(10: 1$, v/v) solvent system. The spots were detected by exposure to a UV lamp at $254 \mathrm{~nm}$. Elemental analyses of obtained compounds were carried out with AMZ 851 CHX analyser (PG, Gdańsk, Poland). The results of elemental analyses $(\mathrm{C}, \mathrm{H}, \mathrm{N})$ were within $\pm 0.4 \%$ of the calculated values.

\section{Preparation of thiosemicarbazide derivatives (1-6)}

3-Hydroxy-2-naphthoic hydrazide $(2.02 \mathrm{~g}, 10 \mathrm{mmol})$ was added to the flask with $15 \mathrm{ml}$ of ethanol and heated under reflux until hydrazide was dissolved. Then $11 \mathrm{mmol}$ of appropriate isothiocyanate was added, and the mixture was heated under reflux for $3 \mathrm{~h}$. Subsequently, the content of the flask was left for $24 \mathrm{~h}$. The obtained precipitate was filtered off, dried and crystallized from ethanol.

\section{$N$-Ethyl-2-[(3-hydroxynaphthalen-2-yl)carbonyl]hydrazi- necarbothioamide (1)}

CAS Registry Number 219538-16-6. Yield: $95 \%$; M.p.: 196-198 ${ }^{\circ} \mathrm{C}$. Analysis for $\mathrm{C}_{14} \mathrm{H}_{15} \mathrm{~N}_{3} \mathrm{O}_{2} \mathrm{~S}$ (289.35) Calculated: C: $58.11 \%, \mathrm{H}: 5.23 \%, \mathrm{~N}: 14.52 \%$; Found: C: $58.22 \%$, H: $5.26 \%$, N: $14.49 \%$. ${ }^{1} \mathrm{H}$ NMR (DMSO- $d_{6}$ ) $\delta$ $(\mathrm{ppm})=1.07-1.11\left(\mathrm{t}, 3 \mathrm{H}, \mathrm{CH}_{3}\right), 3.44-3.50\left(\mathrm{q}, 2 \mathrm{H}, \mathrm{CH}_{2}\right.$, $J=6 \mathrm{~Hz}), 7.32-7.39(\mathrm{~m}, 2 \mathrm{H}, \mathrm{ArH}), 7.50-7.54(\mathrm{~m}, 1 \mathrm{H}$, $\mathrm{ArH}), 7.75-7.78(\mathrm{~d}, 2 \mathrm{H}, \mathrm{ArH}, J=9 \mathrm{~Hz}), 7.87-7.90(\mathrm{~d}, 2 \mathrm{H}$, ArH, $J=9 \mathrm{~Hz}), 8.12-8.14(\mathrm{~m}, 1 \mathrm{H}, \mathrm{ArH}), 8.50(\mathrm{~s}, 1 \mathrm{H}, \mathrm{OH})$, $9.54(\mathrm{~s}, 1 \mathrm{H}, \mathrm{NH}), 10.71(\mathrm{~s}, 1 \mathrm{H}, \mathrm{NH}), 11.51(\mathrm{~s}, 1 \mathrm{H}, \mathrm{NH})$; ${ }^{13} \mathrm{C}$ NMR (DMSO) $\delta(\mathrm{ppm})=14.9\left(\mathrm{CH}_{3}\right), 41.1\left(\mathrm{CH}_{2}\right)$, 111.0, 119.1, 124.3, 126.3, 127.1, 128.9, 129.2, 130.9, $136.4\left(10 \mathrm{C}_{\mathrm{ar}}\right), 154.8(\mathrm{C}=\mathrm{O}), 181.3(\mathrm{C}=\mathrm{S})$.

2-[(3-Hydroxynaphthalen-2-yl) carbonyl]-N-(prop-2-en-1-yl)hydrazinecarbothioamide (2)

CAS Registry Number 224456-14-8. Yield: $80 \%$; M.p.: 197-199 ${ }^{\circ} \mathrm{C}$. Analysis for $\mathrm{C}_{15} \mathrm{H}_{15} \mathrm{~N}_{3} \mathrm{O}_{2} \mathrm{~S}$ (301.36) Calculated: C: $59.78 \%, \mathrm{H}: 5.02 \%, \mathrm{~N}: 13.94 \%$; Found: C: $59.85 \%, \mathrm{H}: 5.05 \%, \mathrm{~N}: 13.90 \% .{ }^{1} \mathrm{H}$ NMR (DMSO- $\left.d_{6}\right) \delta$ $(\mathrm{ppm})=4.12-4.15\left(\mathrm{~m}, 2 \mathrm{H}, \mathrm{CH}_{2}\right), 5.05-5.20\left(\mathrm{~m}, 2 \mathrm{H}, \mathrm{CH}_{2}\right)$, 5.79-5.91 (m, 1H, CH), 7.32-7.39 (m, 2H, ArH), 7.49-7.55 $(\mathrm{m}, 1 \mathrm{H}, \mathrm{ArH}), 7.75-7.78(\mathrm{~d}, 1 \mathrm{H}, \mathrm{ArH}, J=9 \mathrm{~Hz}), 7.87-7.90$ (d, $1 \mathrm{H}, \mathrm{ArH}, J=9 \mathrm{~Hz}), 8.31-8.34(\mathrm{~m}, 1 \mathrm{H}, \mathrm{ArH}), 8.51$ (s, $1 \mathrm{H}, \mathrm{OH}), 9.63(\mathrm{~s}, 1 \mathrm{H}, \mathrm{NH}), 10.77(\mathrm{~s}, 1 \mathrm{H}, \mathrm{NH}), 11.49$ (s, $1 \mathrm{H}, \mathrm{NH}) ;{ }^{13} \mathrm{C}$ NMR (DMSO) $\delta(\mathrm{ppm})=31.2\left(\mathrm{CH}_{2}\right), 46.4$ $\left(\mathrm{CH}_{2}\right), 111.0,115.8,119.0,124.3126 .3,127.1,128.9$, $129.2,130.9,135.3,136.5\left(10 \mathrm{C}_{\mathrm{ar}}\right), 132.6(\mathrm{CH}), 165.1$ $(\mathrm{C}=\mathrm{O}), 183.9(\mathrm{C}=\mathrm{S})$. 
2-[(3-Hydroxynaphthalen-2-yl)carbonyl]-N-(2-methoxyphenyl)hydrazinecarbothioamide (3)

Yield: $94 \%$; M.p.: $196-197{ }^{\circ} \mathrm{C}$. Analysis for $\mathrm{C}_{19} \mathrm{H}_{17} \mathrm{~N}_{3} \mathrm{O}_{3} \mathrm{~S}$ (367.42) Calculated: C: $62.11 \%, \mathrm{H}: 4.66 \%, \mathrm{~N}: 11.44 \%$; Found: C: $62.15 \%, \mathrm{H}: 4.63 \%$, N: $11.47 \% .{ }^{1} \mathrm{H}$ NMR $\left(\mathrm{DMSO}_{6}\right) \delta(\mathrm{ppm})=3.83\left(\mathrm{~s}, 3 \mathrm{H}, \mathrm{CH}_{3}\right), 6.92-6.97(\mathrm{~m}$, 1H, ArH), 7.05-7.07 (m, 1H, ArH), 7.14-7.19 (m, 1H, ArH), 7.32 (s, 1H, OH), 7.34-7.39 (m, 1H, ArH), 7.50-7.55 (m, 1H, ArH), 7.76-7.78 (d, 1H, ArH, $J=6 \mathrm{~Hz}), 7.93-7.94$ (m, 1H, ArH), 8.08-8.09 (m, 1H, ArH), 8.47-8.48 (m, 1H, ArH), 8.63-8.64 (m, 1H, ArH), $9.48(\mathrm{~s}, 1 \mathrm{H}, \mathrm{NH}), 11.20$ (s, 1H, NH), $11.71(\mathrm{~s}, 1 \mathrm{H}, \mathrm{NH}) ;{ }^{13} \mathrm{C}$ NMR (DMSO) $\delta$ $(\mathrm{ppm})=56.18\left(\mathrm{CH}_{3}\right), 111.8,113.3,120.3,121.6,122.5$, $124.3,126.3,128.8,130.2,132.5,136.3,150.1,155.2$ $\left(16 \mathrm{C}_{\mathrm{ar}}\right), 165.1(\mathrm{C}=\mathrm{O}), 180.8(\mathrm{C}=\mathrm{S})$.

\section{2-[(3-Hydroxynaphthalen-2-yl)carbonyl]-N-(3-methoxy-} phenyl)hydrazinecarbothioamide (4)

Yield: $93 \%$; M.p.: $198-200{ }^{\circ} \mathrm{C}$. Analysis for $\mathrm{C}_{19} \mathrm{H}_{17} \mathrm{~N}_{3} \mathrm{O}_{3} \mathrm{~S}$ (367.42) Calculated: C: $62.11 \%, \mathrm{H}: 4.66 \%, \mathrm{~N}: 11.44 \%$; Found: C: $62.18 \%, \mathrm{H}: 4.62 \%, \mathrm{~N}: 11.41 \%$. ${ }^{1} \mathrm{H}$ NMR $\left(\right.$ DMSO- $\left.d_{6}\right) \delta(\mathrm{ppm})=3.75\left(\mathrm{~s}, 3 \mathrm{H}, \mathrm{CH}_{3}\right), 6.73-6.77(\mathrm{~m}$, 2H, ArH), 7.06-7.09 (d, 1H, ArH, $J=9 \mathrm{~Hz}), 7.23-7.28$ $(\mathrm{m}, 2 \mathrm{H}, \mathrm{ArH}), 7.33(\mathrm{~s}, 1 \mathrm{H}, \mathrm{OH}), 7.34-7.39(\mathrm{~m}, 2 \mathrm{H}$, ArH), 7.49-7.55 (m, 1H, ArH), 7.76-7.79 (d, 1H, ArH, $J=9 \mathrm{~Hz}), 7.90-7.93(\mathrm{~m}, 1 \mathrm{H}, \mathrm{ArH}), 8.53(\mathrm{~s}, 1 \mathrm{H}, \mathrm{NH})$, $9.86(\mathrm{~s}, 1 \mathrm{H}, \mathrm{NH}), 11.56(\mathrm{~s}, 1 \mathrm{H}, \mathrm{NH}) ;{ }^{13} \mathrm{C}$ NMR (DMSO) $\delta(\mathrm{ppm})=55.56\left(\mathrm{CH}_{3}\right), 110.9,124.4,121.6,126.3,128.9$, $129.3,130.4,132.5,136.4,140.6,155.2\left(16 \mathrm{C}_{\mathrm{ar}}\right), 165.1$ $(\mathrm{C}=\mathrm{O}), 179.4(\mathrm{C}=\mathrm{S})$.

2-[(3-Hydroxynaphthalen-2-yl)carbonyl]-N-(4-methoxyphenyl)hydrazinecarbothioamide (5)

CAS Registry Number 224456-22-8. Yield: $86 \%$; M.p.: 194-196 ${ }^{\circ} \mathrm{C}$. Analysis for $\mathrm{C}_{19} \mathrm{H}_{17} \mathrm{~N}_{3} \mathrm{O}_{3} \mathrm{~S}$ (367.42) Calculated: C: $62.11 \%, \mathrm{H}: 4.66 \%, \mathrm{~N}: 11.44 \%$; Found: C: $62.15 \%, \mathrm{H}: 4.63 \%, \mathrm{~N}: 11.48 \% .{ }^{1} \mathrm{H}$ NMR (DMSO$\left.d_{6}\right) \delta(\mathrm{ppm})=3.75\left(\mathrm{~s}, 3 \mathrm{H}, \mathrm{CH}_{3}\right), 6.90-6.93(\mathrm{~d}, 2 \mathrm{H}, \mathrm{ArH}$, $J=9 \mathrm{~Hz}), 7.27-7.36(\mathrm{~m}, 3 \mathrm{H}, \mathrm{ArH}), 7.37(\mathrm{~s}, 1 \mathrm{H}, \mathrm{OH})$, 7.47-7.54 (m, 1H, ArH), 7.75-7.78 (d, 2H, ArH, $J=9 \mathrm{~Hz})$, 7.81-7.92 (m, 2H, ArH), $8.53(\mathrm{~s}, 1 \mathrm{H}, \mathrm{NH}), 9.76(\mathrm{~s}, 1 \mathrm{H}$, $\mathrm{NH}), 11.54(\mathrm{~s}, 1 \mathrm{H}, \mathrm{NH}) ;{ }^{13} \mathrm{C}$ NMR $(\mathrm{DMSO}) \delta(\mathrm{ppm})=55.7$ $\left(\mathrm{CH}_{3}\right), 110.9,113.8,119.3,124.3,126.3,127.2,128.9$, $129.3,132.3,136.4\left(16 C_{a r}\right), 157.2(C=O), 179.4(C=S)$.

$N$-(4-Ethoxyphenyl)-2-[(3-hydroxynaphthalen-2-yl)carbonyl]hydrazinecarbothioamide (6)

Yield: $94 \%$; M.p.: $195-197{ }^{\circ} \mathrm{C}$. Analysis for $\mathrm{C}_{20} \mathrm{H}_{19} \mathrm{~N}_{3} \mathrm{O}_{3} \mathrm{~S}$ (381.45) Calculated: C: $62.97 \%, \mathrm{H}$ :
$5.02 \%, \mathrm{~N}: 11.02 \%$; Found: C: $62.91 \%, \mathrm{H}: 5.06 \%, \mathrm{~N}$ : $11.05 \% .{ }^{1} \mathrm{H}$ NMR (DMSO- $\left.d_{6}\right) \delta(\mathrm{ppm})=1.28-1.32(\mathrm{t}$, $\left.3 \mathrm{H}, \mathrm{CH}_{3}, J=6 \mathrm{~Hz}\right), 3.93-4.00\left(\mathrm{q}, 2 \mathrm{H}, \mathrm{CH}_{2}, J=6 \mathrm{~Hz}\right)$, 6.83-6.86 (d, 2H, ArH, $J=9 \mathrm{~Hz}), 7.33-7.36(\mathrm{~d}, 2 \mathrm{H}, \mathrm{ArH}$, $J=9 \mathrm{~Hz}), 7.38-7.39(\mathrm{~m}, 2 \mathrm{H}, \mathrm{ArH}), 7.50-7.55(\mathrm{~m}, 1 \mathrm{H}$, ArH), 7.76-7.79 (d, 1H, ArH, $J=9 \mathrm{~Hz}), 7.88-7.91(\mathrm{~d}$, $1 \mathrm{H}, \mathrm{ArH}, J=9 \mathrm{~Hz}), 8.39(\mathrm{~s}, 1 \mathrm{H}, \mathrm{ArH}), 8.52(\mathrm{~s}, 1 \mathrm{H}, \mathrm{OH})$, $8.76(\mathrm{~s}, 1 \mathrm{H}, \mathrm{NH}), 10.55(\mathrm{~s}, 1 \mathrm{H}, \mathrm{NH}), 11.51(\mathrm{~s}, 1 \mathrm{H}, \mathrm{NH})$; ${ }^{13} \mathrm{C}$ NMR (DMSO) $\delta(\mathrm{ppm})=15.1\left(\mathrm{CH}_{3}\right), 63.7\left(\mathrm{CH}_{2}\right)$, $109.6,114.5,117.0,124.0,126.3,127.2,127.3,128.1$, $128.6,129.6,132.7,135.8,150.3,153.4\left(16 \mathrm{C}_{\mathrm{ar}}\right), 158.8$ $(\mathrm{C}=\mathrm{O}), 168.4(\mathrm{C}=\mathrm{S})$.

\section{Preparation of 4,5-disubstituted-2,4-di- hydro-3H-1,2,4-triazole-3-thiones (7-12)}

A mixture of appropriate thiosemicarbazide 1-6 (10 mmol) and $20-40 \mathrm{ml}$ of $2 \%$ aqueous solution of sodium hydroxide was refluxed for $2 \mathrm{~h}$. Then, the solution was neutralized with diluted hydrochloric acid, and the formed precipitate was filtered off and crystallized from ethanol.

4-Ethyl-5-(3-hydroxynaphthalen-2-yl)-2,4-dihydro-3H-1,2,4-triazole-3-thione (7)

CAS Registry Number 219538-20-2. Yield: $92 \%$; M.p.: 223-225 ${ }^{\circ} \mathrm{C}$. Analysis for $\mathrm{C}_{14} \mathrm{H}_{13} \mathrm{~N}_{3} \mathrm{OS}$ (271.34) Calculated: C: $61.97 \%, \mathrm{H}: 4.83 \%, \mathrm{~N}: 15.49 \%$; Found: C: $61.92 \%, \mathrm{H}: 4.87 \%, \mathrm{~N}: 15.53 \% .{ }^{1} \mathrm{H}$ NMR (DMSO- $d_{6}$ ) $\delta$ $(\mathrm{ppm})=1.03-1.07\left(\mathrm{t}, 3 \mathrm{H}, \mathrm{CH}_{3}, J=6 \mathrm{~Hz}\right), 3.86-3.94(\mathrm{q}$, $\left.2 \mathrm{H}, \mathrm{CH}_{2}, J=9 \mathrm{~Hz}\right), 7.34-7.39$ (m, 2H, ArH), 7.49-7.54 (m, 1H, ArH), 7.79-7.81 (d, 2H, ArH, $J=6 \mathrm{~Hz}), 7.88$ 7.90 (d, 2H, ArH, $J=6 \mathrm{~Hz}), 8.03(\mathrm{~s}, 1 \mathrm{H}, \operatorname{ArH}), 10.65$ (s, 1H, OH), $13.93(\mathrm{~s}, 1 \mathrm{H}, \mathrm{NH}) ;{ }^{13} \mathrm{C}$ NMR (DMSO) $\delta$ $(\mathrm{ppm})=13.7\left(\mathrm{CH}_{3}\right), 45.2\left(\mathrm{CH}_{2}\right), 110.1,116.7,124.2$, $126.5,127.5,128.3,128.7,132.6,136.0,150.0\left(10 \mathrm{C}_{\mathrm{ar}}\right)$, $166.7(\mathrm{C}=\mathrm{S})$.

5-(3-Hydroxynaphthalen-2-yl)-4-(prop-2-en-1-yl)-2,4-dihydro-3H-1,2,4-triazole-3-thione (8)

CAS Registry Number 29213-07-8 Yield: $92 \%$; M.p.: 199-200 ${ }^{\circ} \mathrm{C}$. Analysis for $\mathrm{C}_{15} \mathrm{H}_{13} \mathrm{~N}_{3} \mathrm{OS}$ (283.30) Calculated: C: $63.58 \%, \mathrm{H}: 4.62 \%, \mathrm{~N}: 14.83 \%$; Found: C: $63.52 \%, \mathrm{H}: 4.65 \%$, N: $14.79 \% .{ }^{1} \mathrm{H}$ NMR (DMSO- $d_{6}$ ) $\delta$ $(\mathrm{ppm})=4.61-4.62\left(\mathrm{~d}, 2 \mathrm{H}, \mathrm{CH}_{2}, J=3 \mathrm{~Hz}\right), 4.69-4.95(\mathrm{dd}$, $\left.2 \mathrm{H}, \mathrm{CH}_{2}, J=9 \mathrm{~Hz}\right), 5.59-5.71(\mathrm{~m}, 1 \mathrm{H}, \mathrm{CH}), 7.32-7.37$ (m, 2H, ArH), 7.47-7.53 (m, 1H, ArH), 7.77-7.80 (d, 1H, $\mathrm{ArH}, J=9 \mathrm{~Hz}), 7.86-7.88(\mathrm{~d}, 2 \mathrm{H}, \mathrm{ArH}, J=6 \mathrm{~Hz}), 7.98(\mathrm{~s}$, $1 \mathrm{H}, \mathrm{ArH}), 10.68$ (s, 1H, OH), 14.01 (s, 1H, NH); ${ }^{13} \mathrm{C} \mathrm{NMR}$ $(\mathrm{DMSO}) \delta(\mathrm{ppm})=46.18\left(\mathrm{CH}_{2}\right), 110.1,116.6,124.2$, $126.5,127.5,128.3,128.7,131.9,136.0,150.3\left(10 \mathrm{C}_{\mathrm{ar}}\right)$ $117.6\left(=\mathrm{CH}_{2}\right), 132.6(\mathrm{CH}), 167.4(\mathrm{C}=\mathrm{S})$. 
5-(3-Hydroxynaphthalen-2-yl)-4-(2-methoxyphenyl)-2,4-dihydro-3H-1,2,4-triazole-3-thione (9)

Yield: $93 \%$; M.p.: $220-222{ }^{\circ} \mathrm{C}$. Analysis for $\mathrm{C}_{19} \mathrm{H}_{15} \mathrm{~N}_{3} \mathrm{O}_{2} \mathrm{~S}$ (349.41) Calculated: C: $65.31 \%, \mathrm{H}: 4.33 \%, \mathrm{~N}: 12.03 \%$; Found: C: $65.38 \%, \mathrm{H}: 4.31 \%, \mathrm{~N}: 12.06 \% .{ }^{1} \mathrm{H}$ NMR $\left(\mathrm{DMSO}_{6}\right) \delta(\mathrm{ppm})=3.54\left(\mathrm{~s}, 3 \mathrm{H}, \mathrm{CH}_{3}\right), 6.92-6.99(\mathrm{~m}$, $2 \mathrm{H}, \mathrm{ArH}), 7.05$ (s, 1H, ArH), 7.25-7.35 (m, 3H, ArH), 7.39-7.45 (m, 1H, ArH), 7.62-7.65 (d, 2H, ArH, $J=9 \mathrm{~Hz})$, $7.79-7.81(\mathrm{~d}, 2 \mathrm{H}, \mathrm{ArH}, J=6 \mathrm{~Hz}), 7.87(\mathrm{~s}, 1 \mathrm{H}, \mathrm{ArH})$, 10.19 (s, 1H, OH), 14.00 (s, 1H, NH); ${ }^{13} \mathrm{C}$ NMR (DMSO) $\delta(\mathrm{ppm})=55.9\left(\mathrm{CH}_{3}\right), 109.4,112.8,116.8,120.5,123.0$, $123.9,126.2,127.0,128.0,128.5,130.8,131.3,132.3$, $135.7,150.8,153.5\left(16 \mathrm{C}_{\mathrm{ar}}\right), 168.7(\mathrm{C}=\mathrm{S})$.

5-(3-Hydroxynaphthalen-2-yl)-4-(3-methoxyphenyl)-2,4-dihydro-3H-1,2,4-triazole-3-thione (10)

Yield: $94 \%$; M.p.: $271-273{ }^{\circ} \mathrm{C}$. Analysis for $\mathrm{C}_{19} \mathrm{H}_{15} \mathrm{~N}_{3} \mathrm{O}_{2} \mathrm{~S}$ (349.41) Calculated: C: $65.31 \%$, H: $4.33 \%$, N: $12.03 \%$; Found: C: $65.37 \%, \mathrm{H}: 4.37 \%, \mathrm{~N}: 12.00 \% .{ }^{1} \mathrm{H}$ NMR $\left(\mathrm{DMSO}-d_{6}\right) \delta(\mathrm{ppm})=3.56\left(\mathrm{~s}, 3 \mathrm{H}, \mathrm{CH}_{3}\right), 6.82-6.95(\mathrm{~m}$, $3 \mathrm{H}, \mathrm{ArH}), 7.09$ (s, 1H, ArH), 7.20-7.33 (m, 2H, ArH), 7.42-7.47 (m, 1H, ArH), 7.66-7.68 (d, 1H, ArH, $J=6 \mathrm{~Hz})$, 7.78-7.81 (d, 1H, ArH, $J=9 \mathrm{~Hz}), 8.03(\mathrm{~s}, 1 \mathrm{H}, \mathrm{ArH}), 10.30$ (s, 1H, OH), $14.15(\mathrm{~s}, 1 \mathrm{H}, \mathrm{NH}) ;{ }^{13} \mathrm{C}$ NMR (DMSO) $\delta$ $(\mathrm{ppm})=55.65\left(\mathrm{CH}_{3}\right), 109.6,114.5,116.9,120.4,124.0$, $126.4,127.2,128.1,128.6,129.8,132.7,135.7,135.9$, $150.0,153.5,159.3\left(16 \mathrm{C}_{\mathrm{ar}}\right), 169.1(\mathrm{C}=\mathrm{S})$.

5-(3-Hydroxynaphthalen-2-yl)-4-(4-methoxyphenyl)-2,4-dihydro-3H-1,2,4-triazole-3-thione (11)

CAS Registry Number 910875-05-7. Yield: 95 \%; M.p.: 272-274 ${ }^{\circ} \mathrm{C}$. Analysis for $\mathrm{C}_{19} \mathrm{H}_{15} \mathrm{~N}_{3} \mathrm{O}_{2} \mathrm{~S}$ (349.41) Calculated: C: $65.31 \%, \mathrm{H}: 4.33 \%, \mathrm{~N}: 12.03 \%$; Found: C: $65.37 \%, \mathrm{H}: 4.31 \%, \mathrm{~N}: 12.07 \% .{ }^{1} \mathrm{H}$ NMR (DMSO- $d_{6}$ ) $\delta(\mathrm{ppm})=3.68\left(\mathrm{~s}, 3 \mathrm{H}, \mathrm{CH}_{3}\right), 6.85-6.88(\mathrm{~d}, 2 \mathrm{H}, \mathrm{ArH}$, $J=9 \mathrm{~Hz}), 7.08(\mathrm{~s}, 1 \mathrm{H}, \mathrm{ArH}), 7.19-7.22$ (d, 2H, ArH, $J=9 \mathrm{~Hz}), 7.28-7.33(\mathrm{~m}, 1 \mathrm{H}, \mathrm{ArH}), 7.41-7.46(\mathrm{~m}, 1 \mathrm{H}$, ArH), 7.65-7.68 (d, 1H, ArH, $J=9 \mathrm{~Hz}), 7.79-7.81$ (d, $1 \mathrm{H}, \mathrm{ArH}, J=6 \mathrm{~Hz}), 8.02$ (s, 1H, ArH), $10.27(\mathrm{~s}, 1 \mathrm{H}, \mathrm{OH})$, $14.09(\mathrm{~s}, 1 \mathrm{H}, \mathrm{NH}) ;{ }^{13} \mathrm{C}$ NMR (DMSO) $\delta(\mathrm{ppm})=55.7$ $\left(\mathrm{CH}_{3}\right), 109.6,114.2,116.9,124.0,126.3,127.2,127.4$, $128.1,128.6,129.6,132.7,135.8,150.3,153.4,159.5$ $\left(16 \mathrm{C}_{\mathrm{ar}}\right), 168.4(\mathrm{C}=\mathrm{S})$.

4-(4-Ethoxyphenyl)-5-(3-hydroxynaphthalen-2-yl)-2,4-dihydro-3H-1,2,4-triazole-3-thione (12)

Yield: $94 \%$; M.p.: 274-276 ${ }^{\circ} \mathrm{C}$. Analysis for $\mathrm{C}_{20} \mathrm{H}_{17} \mathrm{~N}_{3} \mathrm{O}_{2} \mathrm{~S}$ (363.43) Calculated: C: $66.10 \%, \mathrm{H}: 4.71 \%$, N: $11.56 \%$; Found: C: $66.15 \%, \mathrm{H}: 4.70 \%, \mathrm{~N}: 11.59 \% .{ }^{1} \mathrm{H}$ NMR $\left(\right.$ DMSO- $\left.d_{6}\right) \delta(\mathrm{ppm})=1.23-1.29\left(\mathrm{t}, 3 \mathrm{H}, \mathrm{CH}_{3}, J=9 \mathrm{~Hz}\right)$, 3.90-3.97 (q, $\left.2 \mathrm{H}, \mathrm{CH}_{2}, J=6 \mathrm{~Hz}, J=9 \mathrm{~Hz}\right), 6.83-6.86(\mathrm{~d}$, $2 \mathrm{H}, \mathrm{ArH}, J=9 \mathrm{~Hz}), 7.07$ (s, $1 \mathrm{H}, \mathrm{ArH}), 7.17-7.20(\mathrm{~d}, 2 \mathrm{H}$, ArH, $J=9 \mathrm{~Hz}), 7.28-7.33(\mathrm{~m}, 1 \mathrm{H}, \mathrm{ArH}), 7.41-7.47(\mathrm{~m}$, $1 \mathrm{H}, \mathrm{ArH}), 7.65-7.68(\mathrm{~d}, 1 \mathrm{H}, \mathrm{ArH}, J=9 \mathrm{~Hz}), 7.79-7.81(\mathrm{~d}$, $1 \mathrm{H}, \mathrm{ArH}, J=6 \mathrm{~Hz}$ ), 8.02 (s, 1H, ArH), $14.08(\mathrm{~s}, 1 \mathrm{H}, \mathrm{NH})$; ${ }^{13} \mathrm{C}$ NMR (DMSO) $\delta(\mathrm{ppm})=15.0\left(\mathrm{CH}_{3}\right), 63.7\left(\mathrm{CH}_{2}\right)$, $109.6,114.5,117.0,124.0,126.3,127.2,127.3,128.1$, $128.6,129.6,132.7,135.8,150.3,153.3,153.4,158.8$ $\left(16 \mathrm{C}_{\mathrm{ar}}\right), 168.4(\mathrm{C}=\mathrm{S})$.

\section{Microbiology}

In vitro antimicrobial assay

The examined compounds $\mathbf{1 - 1 2}$ were screened in vitro for antibacterial and antifungal activity. Procedure for antimicrobial assay is presented in Supplemental Material.

\section{Results and discussion}

\section{Chemistry}

The main purpose of this work was the synthesis, spectral identification and in vitro antimicrobial screening of thiosemicarbazide (1-6) and 1,2,4-triazole-3-thione derivatives (7-12). The compounds 1-12 were synthesized according to the steps presented in Scheme 1. Compounds 3, 4, 6, 9, $\mathbf{1 0}$ and $\mathbf{1 2}$ are unknown in scientific literature.

1,2,4-Triazole derivatives (7-12) were prepared in twostep synthesis. In the first step of synthesis, 3-hydroxy2-naphthoic hydrazide was subjected to condensation reaction with appropriate isothiocyanates to afford 1,4-disubstituted thiosemicarbazide derivatives (1-6). Then new 1,2,4-triazole-3-thiones (7-12) were synthesized by the cyclization reaction of corresponding thiosemicarbazide (1-6) derivatives in alkaline media.

All synthesized compounds are stable solids and dissolve in DMSO in room temperature. The spectral data $\left({ }^{1} \mathrm{H}\right.$ NMR, ${ }^{13} \mathrm{C}$ NMR) confirm the successful synthesis of desired compounds (7-12).

In the ${ }^{1} \mathrm{H}$ NMR spectra of 1,4-disubstituted thiosemicarbazides (1-6), three typical proton singlet signals for the three $\mathrm{NH}$ groups were observed in the range of $\delta 8.53$ $11.71 \mathrm{ppm}$. Whereas in the ${ }^{13} \mathrm{C}$ NMR two signals for $\mathrm{C}=\mathrm{O}$ group and $\mathrm{C}=\mathrm{S}$ group were observed at $\delta 154.8-165.1 \mathrm{ppm}$ and $\delta$ 168.4-183.0 ppm, respectively. The 1,2,4-triazole3-thiones (7-12) in ${ }^{1} \mathrm{H}$ NMR spectra showed characteristic singlet signal for NH group at $\delta 13.93-14.15 \mathrm{ppm}$. Beside this, in ${ }^{13} \mathrm{C}$ NMR spectra for these compounds (7-12) a signal for $\mathrm{C}=\mathrm{S}$ was observed at $\delta 166.7-169.1 \mathrm{ppm}$. All other aliphatic and aromatic signals were found at usual regions. 


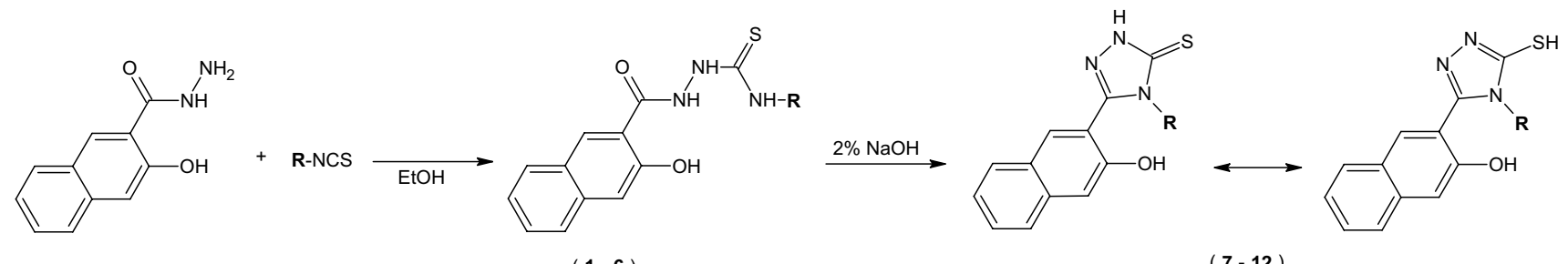

$(1-6)$

$(7-12)$

\begin{tabular}{|c|c|}
\hline Compound No & $\mathbf{R}$ \\
\hline 1,7 & $\mathrm{C}_{2} \mathrm{H}_{5}$ \\
\hline 2,8 & $\mathrm{CH}_{2}=\mathrm{CHCH}_{2}$ \\
\hline 3,9 & $2-\mathrm{CH}_{3} \mathrm{OC}_{6} \mathrm{H}_{4}$ \\
\hline 4,10 & $3-\mathrm{CH}_{3} \mathrm{OC}_{6} \mathrm{H}_{4}$ \\
\hline 5,11 & $4-\mathrm{CH}_{3} \mathrm{OC}_{6} \mathrm{H}_{4}$ \\
\hline 6,12 & $4-\mathrm{CH}_{3} \mathrm{CH}_{2} \mathrm{OC}_{6} \mathrm{H}_{4}$ \\
\hline
\end{tabular}

Scheme 1 Synthesis scheme of 1,2,4-triazole derivatives (7-12)

Table 1 Activity data expressed as MIC $[\mu \mathrm{g} / \mathrm{ml}]$ against the reference strains of bacteria and fungi

\begin{tabular}{|c|c|c|c|c|c|c|c|c|c|c|c|c|c|}
\hline \multirow[t]{2}{*}{ Species/compound } & \multicolumn{9}{|c|}{ MIC (MBC/MFC) $[\mu \mathrm{g} / \mathrm{ml}]$ of the tested compounds } & \multirow[t]{2}{*}{ CPX } & \multirow[t]{2}{*}{ CFX } & \multirow[t]{2}{*}{ APC } & \multirow[t]{2}{*}{ FLU } \\
\hline & 1 & 2 & 3 & 4 & 5 & 7 & 8 & 9 & 11 & & & & \\
\hline \multicolumn{14}{|l|}{ Gram-positive bacteria } \\
\hline Staphylococcus aureus ATCC 25923 & 15.62 & 15.62 & - & 15.62 & - & 62.5 & 62.5 & 125 & 250 & 0.488 & 0.49 & nd & nd \\
\hline Staphylococcus aureus ATCC 6538 & 31.25 & 31.25 & - & 15.62 & - & 125 & 125 & 62.5 & 250 & 0.244 & 0.98 & nd & nd \\
\hline Staphylococcus aureus ATCC 43300 & 15.62 & 7.81 & 250 & 7.81 & - & 62.5 & 125 & 31.25 & 250 & 0.244 & nd & nd & nd \\
\hline Staphylococcus epidermidis ATCC 12228 & 7.81 & 3.91 & - & 15.62 & - & 62.5 & 125 & 62.5 & 250 & 0.122 & 0.24 & nd & nd \\
\hline Micrococcus luteus ATCC 10240 & 62.5 & 250 & - & 31.25 & 1000 & 125 & 125 & 31.25 & 125 & 0.976 & 0.98 & nd & nd \\
\hline Bacillus subtilis ATCC 6633 & 31.25 & 15.62 & - & 31.25 & - & 125 & 125 & 62.5 & 250 & 0.031 & 15.62 & nd & nd \\
\hline Bacillus cereus ATCC 10876 & 15.62 & 15.62 & - & 1000 & - & 125 & 125 & 62.5 & 250 & 0.061 & 31.25 & 62.5 & nd \\
\hline \multicolumn{14}{|l|}{ Gram-negative bacteria } \\
\hline Bordetella bronchiseptica ATCC 4617 & - & - & - & 250 & - & 500 & 500 & - & - & 0.976 & nd & nd & nd \\
\hline \multicolumn{14}{|l|}{ Fungi } \\
\hline Candida albicans ATCC 2091 & - & - & - & 1000 & - & 500 & 500 & 1000 & - & nd & nd & nd & 0.244 \\
\hline Candida albicans ATCC 10231 & 1000 & - & - & 1000 & - & 500 & 500 & - & - & nd & nd & nd & 0.976 \\
\hline Candida parapsilosis ATCC 22019 & 1000 & - & - & - & - & 500 & 500 & 1000 & - & nd & nd & nd & 1.953 \\
\hline
\end{tabular}

The standard compounds used as positive controls: ciprofloxacin (CPX), cefuroxime (CFX), ampicillin (APC) for bacteria and fluconazole (FLU*) for fungi

Compounds with bactericidal effect $(\mathrm{MBC} / \mathrm{MIC} \leq 4)$ are marked with bold

“-" no activity, $n d$ not determined, $M I C$ minimal inhibitory concentration, $M B C$ minimal bactericidal concentration

The above mentioned spectral data successfully confirmed the formation of expected products.

The synthesized compounds (1-12) were screened in vitro for antibacterial and antifungal activity.

\section{Microbiology}

The results of our study indicated that examined compounds 1-3, 5, 6, 9-12 had no inhibitory effect on the growth of reference strains of Gram-negative bacteria, i.e., E. coli ATCC 25922, Klebsiella pneumoniae ATCC 13883,
Proteus mirabilis ATCC 12453, Salmonella typhimurium ATCC 14028 and P. aeruginosa ATCC 9027. Moreover, compounds 6, 10 and $\mathbf{1 2}$ had no activity against the other reference strains of bacteria (Table 1).

On the basis of minimal inhibitory concentration (MIC) values presented in Table 1, obtained by the broth microdilution method, it was shown that some of the tested compounds showed high influence on the growth of Gram-positive bacteria.

Among them, compounds 1, $\mathbf{2}$ and $\mathbf{4}$ possessed the highest activity against staphylococci with MIC $=3.91-31.25 \mu \mathrm{g} /$ 
ml. S. epidermidis ATCC 12228 and S. aureus ATCC 43300 were especially sensitive to them.

Compound 1 showed highest activity toward $S$. epidermidis ATCC $12228(\mathrm{MIC}=7.81 \mu \mathrm{g} / \mathrm{ml})$ and S. aureus ATCC 43300 (MIC $=15.62 \mu \mathrm{g} / \mathrm{ml}$ ) with bactericidal effect. The activity against other Staphylococcus species was strong $(\mathrm{MIC}=15.62-31.25 \mu \mathrm{g} / \mathrm{ml})$. Compound 2 showed the highest activity of all synthesized compounds against $S$. epidermidis ATCC 12228 ( $\mathrm{MIC}=3.91 \mu \mathrm{g} / \mathrm{ml}$ ) but with bacteriostatic effect. The activity against $S$. aureus ATCC 43300 was also very strong (MIC $=7.81 \mu \mathrm{g} / \mathrm{ml}$ ) or strong against $S$. aureus ATCC 25923 and S. aureus ATCC 6538 with MIC $=15.62 \mu \mathrm{g} / \mathrm{ml}$ and $31.25 \mu \mathrm{g} / \mathrm{ml}$, respectively. Compound 4 showed the strongest activity with bactericidal effect against $S$. aureus ATCC 43300. Its activity against other staphylococci was also very good (MIC $=15.62 \mu \mathrm{g} / \mathrm{ml}$ ).

The growth of Micrococcus luteus ATCC 10240 was inhibited strongly by compounds 4 and $\mathbf{9}$ (MIC $=31.25 \mu \mathrm{g}$ / $\mathrm{ml})$ with bacteriostatic effect. The MIC values of other compounds were much higher for this microorganism $(\mathrm{MIC}=62.5-125 \mu \mathrm{g} / \mathrm{ml})$, with bacteriostatic or bactericidal effect.

Considering Bacillus subtilis ATCC 6633, the highest antibacterial activity against this microorganism was shown by compound 2 ( $\mathrm{MIC}=15.62 \mu \mathrm{g} / \mathrm{ml}$ ) with bacteriostatic effect. The activity of this compound was equal to the activity of cefuroxime ( $\mathrm{MIC}=15.62 \mu \mathrm{g} / \mathrm{ml}$ ). Compounds $\mathbf{1}$ and $\mathbf{4}$ showed also high activity against this bacterium $(\mathrm{MIC}=31.25 \mu \mathrm{g} / \mathrm{ml})$ with bacteriostatic and bactericidal effect, respectively.

It is worth to underline that compounds $\mathbf{1}$ and $\mathbf{2}$ showed two times higher activity than cefuroxime (MIC $=31.25 \mu \mathrm{g} / \mathrm{ml})$ against Bacillus cereus ATCC 10876. The activity of these compounds (MIC $=15.62 \mu \mathrm{g}$ / $\mathrm{ml}$ ) was also 4 times higher than the activity of ampicillin (MIC $=62.5 \mu \mathrm{g} / \mathrm{ml}$ ) against this pathogen. The sensitiveness of $B$. cereus to synthesized compounds is very important due to the fact that this pathogen is a common source of severe infections [38].

The activity of compounds 7-9 against Gram-positive bacteria was good with MIC $=62.5-125 \mu \mathrm{g} / \mathrm{ml}$, and had bactericidal or bacteriostatic effect $(\mathrm{MBC} / \mathrm{MIC}=1-32)$. In turn, the minimum concentrations of 11, which inhibited the growth of these microorganisms were lower (MIC $=125-250 \mu \mathrm{g} / \mathrm{ml})$.

Besides, compounds 4, 7 and 8 were found to exhibit some activity against B. bronchiseptica ATCC 4617, belonging to Gram-negative bacteria (MIC $=250-500 \mu \mathrm{g}$ / $\mathrm{ml}$ ). Compounds $\mathbf{7}$ and $\mathbf{8}$ had bactericidal effect against this microorganism.

The obtained compounds indicated also some activity against Candida spp. ATCC, belonging to yeasts. The substances $\mathbf{7}$ and $\mathbf{8}$ exhibited moderate activity
(MIC $=500 \mu \mathrm{g} / \mathrm{ml}$ ) with fungicidal effect. The other substances had mild or no activity toward yeasts (Table 1).

\section{Structure-activity relationship}

On the basis of our antimicrobial assay results, we can conclude that the introduction of 3-methoxyphenyl substituent in 1,2,4-triazole derivative (10) can be connected with the disappearance of the activity of this compound, whereas the same substituent in thiosemicarbazide (4) did not revealed such attribute and was active against many bacterial strains.

The thiosemicarbazide derivatives $\mathbf{1}$ and $\mathbf{2}$ substituted with aliphatic substituents: ethyl (1) and allyl (2) as well as compound $\mathbf{4}$ substituted with meta-methoxyphenyl showed the strongest activity among all of synthesized compounds. Ortho-methoxyphenyl and para-methoxyphenyl thiosemicarbazide derivatives $(\mathbf{3}, \mathbf{5})$ showed the weakest activity among all thiosemicarbazide derivatives. In the case of thiosemicarbazide (6) and 1,2,4-triazole derivative (12), the presence of 4-ethoxyphenyl substituent can be connected with the lack of antimicrobial activity. The 1,2,4-triazole derivative 9, which is substituted with ortho-methoxyphenyl substituent in comparison to the thiosemicarbazide with the same substituent (3), showed moderate to good activity against bacterial strains.

In general, the introduction of alkyl $(\mathbf{1}, \mathbf{2})$ and 3-methoxyphenyl substituents (4) was the most beneficial in terms of bioactivity in the case of thiosemicarbazides, whereas in 1,2,4-triazoles the compounds with alkyl $(\mathbf{7}, \mathbf{8}), 2$-methoxyphenyl (9), 4-methoxyphenyl (11) substituents displayed the most significant activity.

\section{Conclusions}

In this work, we synthesized and analyzed 6 thiosemicarbazide derivatives (1-6) and 6 1,2,4-triazole derivatives (7-12) and tested them against a panel of bacterial and fungal strains. Our antimicrobial screening results indicate that some of the newly obtained compounds showed significant antibacterial activity, especially against Gram-positive bacteria. Among them, the activities of compounds 1, 2 and $\mathbf{4}$ were moderate to strong against staphylococci. In addition to these compounds $\mathbf{1}$ and $\mathbf{2}$ showed two times higher activity than cefuroxime and 4 times higher activity than ampicillin against $B$. cereus ATCC 10876.

Acknowledgments Dr Łukasz Popiołek is awarded with the Fellowship for Young Scientists with Outstanding Scientific Achievements from the Medical University of Lublin (Poland).

\section{Compliance with ethical standards}

Conflict of interest The authors declare no conflict of interest. 
Open Access This article is distributed under the terms of the Creative Commons Attribution 4.0 International License (http://creativecommons.org/licenses/by/4.0/), which permits unrestricted use, distribution, and reproduction in any medium, provided you give appropriate credit to the original author(s) and the source, provide a link to the Creative Commons license, and indicate if changes were made.

\section{References}

1. R.C. Moellering Jr., Int. J. Antimicrob. Agents 37(1), 2-9 (2011)

2. S. Singhal, S. Arora, S. Agarwal, R. Sharma, N. Singhal, J. Pharm. Pharm. Sci. 2(6), 4661-4681 (2013)

3. M. Sheikhy, A.R. Jalilian, A. Novinrooz, F. Motamedi-Sedeh, J. Biomed. Sci. Eng. 5, 39-42 (2012)

4. P. Umadevi, K. Deepti, I. Srinath, G. Vijayalakshmi, M. Tarakaramji, Int. J. Pharm. Pharm. Sci. 4(3), 379-383 (2012)

5. M.A.M.S.H. El-Sharief, S.Y. Abbas, K.A.M. El-Bayouki, E.W. El-Gammal, Eur. J. Med. Chem. 67, 263-268 (2013)

6. P.C. Jain, N.P.S. Prakash, M.M.S. Kinthada, M.C. Jain, Int. J. PharmTech. 2(3), 726-734 (2010)

7. Z.C. Li, L.H. Chen, X.J. Yu, Y.H. Hu, K.K. Song, X.W. Zhou et al., J. Agric. Food Chem. 58, 12537-12540 (2010)

8. V. Alagarsamy, P. Parthiban, Rasǎyan J. Chem. 4(4), 547-553 (2011)

9. S. Rastogi, H. Rastogi, Ind. J. Chem. 49B, 547-553 (2010)

10. R.J. Nevagi, A.S. Dhake, H.I. Narkhede, R. Kaur, Bioorg. Chem. 54, 68-72 (2014)

11. H.J. Zhang, Y. Qian, D.D. Zhu, X.G. Yang, H.L. Zhu, Eur. J. Med. Chem. 46, 4702-4708 (2011)

12. S. Padhye, Z. Afrasiabi, E. Sinn, J. Fok, K. Mehta, N. Rath, Inorg. Chem. 44, 1154-1156 (2005)

13. B.S. Patil, G. Krishnamurthy, H.S.B. Naik, P.R. Latthe, M. Ghate, Eur. J. Med. Chem. 45, 3329-3334 (2010)

14. G. Turan-Zitouni, Z.A. Kaplancikli, M.T. Yildiz, P. Chevallet, D. Kaya, Eur. J. Med. Chem. 40, 607-613 (2005)

15. D. Ji, J. Lu, B. Lu, C.H. Xin, J. Mu, J. Li, C.H. Peng, X. Bao, Bioorg. Med. Chem. Lett. 23, 1997-2000 (2013)

16. M. Koparir, C. Orek, A.E. Parlak, A. Soylemez, P. Koparir, M. Karatepe, S.D. Dastan, Eur. J. Med. Chem. 63, 340-346 (2013)

17. Y.J. Mange, A.M. Isloor, S. Malladi, S. Isloor, H.-K. Fun, Arab. J. Chem. 26, 177-181 (2013)
18. R. Singh, G.V. Pujar, M.N. Purohit, V.M. Chandrashekar, Med. Chem. Res. 22, 2163-2173 (2013)

19. H. Bendaha, L. Yu, R. Touzani, R. Souane, G. Giaever, C. Nislow, C.H. Boone, S.E. Kadiri, G.W. Brown, M. Bellaoui, Eur. J. Med. Chem. 46, 4117-4124 (2011)

20. P.Z. Zhang, S.F. Zhou, T.R. Li, J. Jiang, Chin. Chem. Lett. 23, 1381-1384 (2012)

21. Y. Zou, Q. Zhao, J. Liao, H. Hu, S. Yu, X. Chai, M. Xu, Q. Wu, Bioorg. Med. Chem. Lett. 22, 2959-2962 (2012)

22. M. Shiradkar, G.V.S. Kumar, V. Dasari, S. Tatikonda, K.C.H. Akula, R. Shah, Eur. J. Med. Chem. 42, 807-816 (2007)

23. N.B. Patel, I.H. Khan, S.D. Rajani, Eur. J. Med. Chem. 45, 4293-4299 (2010)

24. C.H. Menendez, A. Chollet, F. Rodriguez, C. Inard, M.R. Pasca, C.H. Lherbet, M. Baltas, Eur. J. Med. Chem. 52, 275-283 (2012)

25. K.M. Krishna, B. Inturi, G.V. Pujar, M.N. Purohit, G.S. Vijaykumar, Eur. J. Med. Chem. 84, 516-529 (2014)

26. Y.A. Al-Soud, M.N. Al-Dweri, N.A. Al-Masoudi, Farmaco 59 , 775-783 (2004)

27. Y.A. Al-Soud, N.A. Al-Masoudi, A.E.-R.S. Ferwanah, Bioorg. Med. Chem. 11, 1701-1708 (2003)

28. P.P. Kattimani, R.R. Kamble, M.Y. Kariduraganavar, A. Dorababu, R.K. Hunnur, Eur. J. Med. Chem. 62, 232-240 (2013)

29. G.E.D.A.A. Abuo-Rahma, M. Abdel-Aziz, E.A.M. Beshr, T.F.S. Ali, Eur. J. Med. Chem. 71, 185-198 (2014)

30. A. Krajczyk, K. Kulinska, T. Kulinski, B.L. Hurst, C.W. Day, D.F. Smee, T. Ostrowski, P. Januszczyk, J. Zeidler, Antivir. Chem. Chemother. 23(4), 161-171 (2014)

31. J. Heeres, R. Hendrickx, J.J. Van Cutsem, Med. Chem. 26(4), 611-613 (1983)

32. H. Washton, Diagn. Microbiol. Infect. Dis. 12(4 Suppl), 229-233 (1989)

33. A. Kotiranta, K. Lounatmaa, M. Haapasalo, Microbes Infect. 2, 189-198 (2000)

34. A. Pompella, M. Comporti, Histochemistry 95, 255-262 (1991)

35. H.N. Dogan, S. Rollas, H. Erdeniz, Farmaco 53, 462-467 (1998)

36. A.El-G.M. Khalil, A.M. Berghot, M.A. Gouda, J. Saudi Chem. Soc. 20, 165-172 (2016)

37. H.N. Dogan, A. Duran, S. Rollas, Ind. J. Chem. 44B, 2301-2307 (2005)

38. E.J. Bottone, Clin. Microbiol. Rev. 23, 382-398 (2010) 